\title{
VARIANTS OF ČEBYŠEV'S INEQUALITY WITH APPLICATIONS
}

M. KLARIČIĆ BAKULA, A. MATKOVIĆ, AND J. PEČARIĆ

Received 19 December 2005; Accepted 2 April 2006

Several variants of Čebyšev's inequality for two monotonic $n$-tuples and also $k \geq 3$ nonnegative $n$-tuples monotonic in the same direction are presented. Immediately after that their refinements of Ostrowski's type are given. Obtained results are used to prove generalizations of discrete Milne's inequality and its converse in which weights satisfy conditions as in the Jensen-Steffensen inequality.

Copyright (c) 2006 M. Klaričić Bakula et al. This is an open access article distributed under the Creative Commons Attribution License, which permits unrestricted use, distribution, and reproduction in any medium, provided the original work is properly cited.

\section{Introduction}

In 2003 Mercer gave the following interesting variant of the discrete Jensen's inequality (see, e.g., [8, page 43]) for convex functions.

Theorem 1.1 [4, Theorem 1]. If $f$ is a convex function on an interval containing $n$-tuple $\mathbf{x}=\left(x_{1}, \ldots, x_{n}\right)$ such that $0<x_{1} \leq x_{2} \leq \cdots \leq x_{n}$ and $\mathbf{w}=\left(w_{1}, \ldots, w_{n}\right)$ is positive $n$-tuple with $\sum_{i=1}^{n} w_{i}=1$, then

$$
f\left(x_{1}+x_{n}-\sum_{i=1}^{n} w_{i} x_{i}\right) \leq f\left(x_{1}\right)+f\left(x_{n}\right)-\sum_{i=1}^{n} w_{i} f\left(x_{i}\right) .
$$

Two years later his result was generalized as it is stated below.

Theorem $1.2\left[1\right.$, Theorem 2]. Let $[a, b]$ be an interval in $\mathbb{R}, a<b$. Let $\mathbf{x}=\left(x_{1}, \ldots, x_{n}\right)$ be $a$ monotonic $n$-tuple in $[a, b]^{n}$, and let $\mathbf{w}=\left(w_{1}, \ldots, w_{n}\right)$ be a real $n$-tuple such that

$$
0 \leq W_{k} \leq W_{n}(k=1, \ldots, n-1), \quad W_{n}>0,
$$


2 Variants of Čebyšev's inequality with applications

where $W_{k}=\sum_{i=1}^{k} w_{i}(k=1, \ldots, n)$. If function $f:[a, b] \rightarrow \mathbb{R}$ is convex, then

$$
f\left(a+b-\frac{1}{W_{n}} \sum_{i=1}^{n} w_{i} x_{i}\right) \leq f(a)+f(b)-\frac{1}{W_{n}} \sum_{i=1}^{n} w_{i} f\left(x_{i}\right) .
$$

As we can see, here the condition $w_{i}>0(i=1, \ldots, n)$ is relaxed on the conditions (1.2) as in the well-known Jensen-Steffensen inequality for sums (see, e.g, [8, page 57]).

Remark 1.3. It can be easily proved that for a real $n$-tuple $\mathbf{w}$ which satisfies (1.2) and for any monotonic $n$-tuple $\mathbf{x} \in[a, b]^{n}$ the inequalities

$$
a \leq \frac{1}{W_{n}} \sum_{i=1}^{n} w_{i} x_{i} \leq b,
$$

hold. From (1.4) we can also conclude that $a+b-\frac{1}{W_{n}} \sum_{i=1}^{n} w_{i} x_{i} \in[a, b]$.

In this paper we present "Mercer's type" variants of several well-known inequalities.

In Section 2 we give generalizations of the discrete Čebyšev's inequality for two monotonic $n$-tuples and also for $k \geq 3$ nonnegative $n$-tuples monotonic in the same direction, in which weights $\mathbf{w}$ satisfy the conditions (1.2). Immediately after Mercer's type variants of those inequalities are presented. In Section 3 we give analogous variants of Pečarićs generalizations of the discrete Ostrowski's inequalities. In Section 4 we use results from Section 2 to obtain generalizations of Milne's inequality and its converse. Mercer's type variants of Milne's inequality and its converse are also given.

\section{Variants of Čebyšev's inequality}

A classic result due to Čebyšev $(1882,1883)$ is stated as follows. Let $\mathbf{w}$ be a nonnegative $n$-tuple. If real $n$-tuples $\mathbf{x}=\left(x_{1}, \ldots, x_{n}\right)$ and $\mathbf{y}=\left(y_{1}, \ldots, y_{n}\right)$ are monotonic in the same direction, then

$$
\sum_{i=1}^{n} w_{i} x_{i} \sum_{i=1}^{n} w_{i} y_{i} \leq \sum_{i=1}^{n} w_{i} \sum_{i=1}^{n} w_{i} x_{i} y_{i}
$$

If $\mathbf{x}$ and $\mathbf{y}$ are monotonic in opposite directions, the inequality (2.1) is reversed.

Although the proof of the following generalization of the inequality (2.1) has been already known (see [6]) for the sake of clarity, we will briefly present it here.

TheOREM 2.1. Let $\mathbf{w}=\left(w_{1}, \ldots, w_{n}\right)$ be a real $n$-tuple such that (1.2) is satisfied. Then for any real $n$-tuples $\mathbf{x}=\left(x_{1}, \ldots, x_{n}\right), \mathbf{y}=\left(y_{1}, \ldots, y_{n}\right)$ monotonic in the same direction the inequality (2.1) holds. If $\mathbf{x}$ and $\mathbf{y}$ are monotonic in opposite directions, (2.1) is reversed.

Proof. Using the well-known Abel's identity it can be proved that the following identity holds:

$$
\begin{aligned}
\sum_{i=1}^{n} w_{i} & \sum_{i=1}^{n} w_{i} x_{i} y_{i}-\sum_{i=1}^{n} w_{i} x_{i} \sum_{i=1}^{n} w_{i} y_{i} \\
& =\sum_{k=1}^{n-1}\left[\sum_{l=1}^{k-1} \bar{W}_{k+1} W_{l}\left(x_{l+1}-x_{l}\right)\left(y_{k+1}-y_{k}\right)+\sum_{l=k+1}^{n} \bar{W}_{l} W_{k}\left(x_{l}-x_{l-1}\right)\left(y_{k+1}-y_{k}\right)\right],
\end{aligned}
$$


where $\bar{W}_{k}=\sum_{i=k}^{n} w_{i}$. Suppose that $\mathbf{x}$ and $\mathbf{y}$ are monotonic in the same direction. Then

$$
\left(x_{i+1}-x_{i}\right)\left(y_{j+1}-y_{j}\right) \geq 0
$$

for all $i, j \in\{1, \ldots, n-1\}$. Furthermore, the conditions (1.2) on $n$-tuple w imply that also

$$
\bar{W}_{k} \geq 0 \quad(k=1, \ldots, n),
$$

so from identity (2.2) we may conclude that

$$
\sum_{i=1}^{n} w_{i} \sum_{i=1}^{n} w_{i} x_{i} y_{i}-\sum_{i=1}^{n} w_{i} x_{i} \sum_{i=1}^{n} w_{i} y_{i} \geq 0
$$

If $\mathbf{x}$ and $\mathbf{y}$ are monotonic in opposite directions, we have

$$
\left(x_{i+1}-x_{i}\right)\left(y_{j+1}-y_{j}\right) \leq 0
$$

for all $i, j \in\{1, \ldots, n-1\}$, so the reverse of (2.1) immediately follows.

This completes the proof.

In the next theorem we give a Mercer's type variant of the inequality (2.1).

Theorem 2.2. Let $n \geq 2$ and let $\mathbf{w}$ be a real $n$-tuple such that (1.2) is satisfied. Let $[a, b]$ and $[c, d]$ be intervals in $\mathbb{R}$, where $a<b, c<d$. Then for any real $n$-tuples $\mathbf{x} \in[a, b]^{n}$ and $\mathbf{y} \in[c, d]^{n}$ monotonic in the same direction,

$$
\left(a+b-\frac{1}{W_{n}} \sum_{i=1}^{n} w_{i} x_{i}\right)\left(c+d-\frac{1}{W_{n}} \sum_{i=1}^{n} w_{i} y_{i}\right) \leq a c+b d-\frac{1}{W_{n}} \sum_{i=1}^{n} w_{i} x_{i} y_{i} .
$$

If $\mathbf{x}$ and $\mathbf{y}$ are monotonic in opposite directions, the inequality (2.7) is reversed.

Proof. Without any loss of generality we may suppose that $n$-tuples $\mathbf{x}$ and $\mathbf{y}$ are both monotonically decreasing (in other cases the proof is similar). We define $(n+2)$-tuples $\mathbf{w}^{\prime}=\left(w_{1}^{\prime}, \ldots, w_{n+2}^{\prime}\right), \mathbf{x}^{\prime}=\left(x_{1}^{\prime}, \ldots, x_{n+2}^{\prime}\right)$, and $\mathbf{y}^{\prime}=\left(y_{1}^{\prime}, \ldots, y_{n+2}^{\prime}\right)$ as

$$
\begin{aligned}
& w_{1}^{\prime}=1, w_{2}^{\prime}=-\frac{w_{1}}{W_{n}}, \ldots, w_{n+1}^{\prime}=-\frac{w_{n}}{W_{n}}, \quad w_{n+2}^{\prime}=1, \\
& x_{1}^{\prime}=b, \quad x_{2}^{\prime}=x_{1}, \ldots, x_{n+1}^{\prime}=x_{n}, \quad x_{n+2}^{\prime}=a, \\
& y_{1}^{\prime}=d, \quad y_{2}^{\prime}=y_{1}, \ldots, y_{n+1}^{\prime}=y_{n}, \quad y_{n+2}^{\prime}=c .
\end{aligned}
$$

Obviously, $\mathbf{x}^{\prime}$ and $\mathbf{y}^{\prime}$ are both monotonically decreasing and we have

$$
0 \leq W_{k}^{\prime} \leq 1 \quad(k=1, \ldots, n+1), \quad W_{n+2}^{\prime}=1,
$$

so we may apply Theorem 2.1 on $(n+2)$-tuples $\mathbf{w}^{\prime}, \mathbf{x}^{\prime}$, and $\mathbf{y}^{\prime}$ to obtain

$$
\sum_{i=1}^{n+2} w_{i}^{\prime} x_{i}^{\prime} \sum_{i=1}^{n+2} w_{i}^{\prime} y_{i}^{\prime} \leq \sum_{i=1}^{n+2} w_{i}^{\prime} \sum_{i=1}^{n+2} w_{i}^{\prime} x_{i}^{\prime} y_{i}^{\prime}
$$

from which we can easily get (2.7). 
4 Variants of Čebyšev's inequality with applications

Čebyšev's inequality can be generalized for $k \geq 3$ nonnegative $n$-tuples monotonic in the same direction with nonnegative weights $\mathbf{w}$ (see, e.g., [8, page 198]). Here we give an analogous generalization of Čebyšev's inequality for $k \geq 3$ nonnegative $n$-tuples in which weights w satisfy the conditions (1.2). Partial order " $\leq$ " on $\mathbb{R}^{k}$ here is defined as

$$
\left(x_{1}, \ldots, x_{k}\right) \leq\left(y_{1}, \ldots, y_{k}\right) \Longleftrightarrow x_{1} \leq y_{1} \wedge \cdots \wedge x_{k} \leq y_{k} .
$$

In order to simplify our results, we will consider only weights $\mathbf{w}$ with sum 1 .

Theorem 2.3. Let $n \geq 2$ and let $\mathbf{w}$ be a real $n$-tuple such that

$$
0 \leq W_{k} \leq 1 \quad(k=1, \ldots, n-1), \quad W_{n}=1 .
$$

Let $k \geq 2$ and let $I \subseteq[0,+\infty\rangle^{k}$. Then for any $\mathbf{x}^{(1)}, \ldots, \mathbf{x}^{(n)} \in I$ such that

$$
\mathbf{x}^{(1)} \leq \cdots \leq \mathbf{x}^{(n)} \quad \text { or } \quad \mathbf{x}^{(1)} \geq \cdots \geq \mathbf{x}^{(n)},
$$

the following holds:

$$
\prod_{i=1}^{k} \sum_{j=1}^{n} w_{j} x_{i}^{(j)} \leq \sum_{j=1}^{n} w_{j} \prod_{i=1}^{k} x_{i}^{(j)}
$$

Proof. The proof of (2.14) is by induction on $k$. The case $k=2$ follows from Theorem 2.1. Suppose that (2.14) is valid for all $l, 2 \leq l \leq k$. We have

$$
\sum_{j=1}^{n} w_{j} \prod_{i=1}^{k+1} x_{i}^{(j)}=\sum_{j=1}^{n} w_{j} \prod_{i=1}^{k} x_{i}^{(j)} x_{k+1}^{(j)}
$$

and we know that

$$
\prod_{i=1}^{k} \sum_{j=1}^{n} w_{j} x_{i}^{(j)} \geq 0, \quad \sum_{j=1}^{n} w_{j} \prod_{i=1}^{k} x_{i}^{(j)} \geq 0, \quad \sum_{j=1}^{n} w_{j} x_{k+1}^{(j)} \geq 0
$$

(see Remark 1.3). We define nonnegative $n$-tuple $\mathbf{y}$ as

$$
y_{j}=\prod_{i=1}^{k} x_{i}^{(j)} \quad(j=1, \ldots, n) .
$$

It can be easily seen that $\mathbf{y}$ is monotonic in the same sense as $\left(\mathbf{x}^{(1)}, \ldots, \mathbf{x}^{(n)}\right)$, that is, $\mathbf{y}$ is monotonic in the same sense as $\left(x_{k+1}^{(1)}, \ldots, x_{k+1}^{(n)}\right)$, so we may apply $(2.1)$ and our induction hypothesis in (2.15) to obtain

$$
\begin{aligned}
\sum_{j=1}^{n} w_{j} \prod_{i=1}^{k+1} x_{i}^{(j)} & =\sum_{j=1}^{n} w_{j} \prod_{i=1}^{k} x_{i}^{(j)} x_{k+1}^{(j)} \geq\left(\sum_{j=1}^{n} w_{j} \prod_{i=1}^{k} x_{i}^{(j)}\right)\left(\sum_{j=1}^{n} w_{j} x_{k+1}^{(j)}\right) \\
& \geq\left(\prod_{i=1}^{k} \sum_{j=1}^{n} w_{j} x_{i}^{(j)}\right)\left(\sum_{j=1}^{n} w_{j} x_{k+1}^{(j)}\right)=\prod_{i=1}^{k+1} \sum_{j=1}^{n} w_{j} x_{i}^{(j)},
\end{aligned}
$$

so by induction the result holds. 
In the next theorem we give a Mercer's type variant of (2.14).

Theorem 2.4. Let $n \geq 2$ and let $\mathbf{w}$ be a real $n$-tuple such that (2.12) is satisfied. Let $k \geq 2$ and let $I=\left[a_{1}, b_{1}\right] \times \cdots \times\left[a_{k}, b_{k}\right] \subset[0,+\infty\rangle^{k}$. Then for any $\mathbf{x}^{(1)}, \ldots, \mathbf{x}^{(n)} \in I$ such that

$$
\mathbf{x}^{(1)} \leq \cdots \leq \mathbf{x}^{(n)} \quad \text { or } \quad \mathbf{x}^{(1)} \geq \cdots \geq \mathbf{x}^{(n)},
$$

the following holds:

$$
\prod_{i=1}^{k}\left(a_{i}+b_{i}-\sum_{j=1}^{n} w_{j} x_{i}^{(j)}\right) \leq \prod_{i=1}^{k} a_{i}+\prod_{i=1}^{k} b_{i}-\sum_{j=1}^{n} w_{j} \prod_{i=1}^{k} x_{i}^{(j)} .
$$

Proof. Suppose that $\mathbf{x}^{(1)} \leq \cdots \leq \mathbf{x}^{(n)}$. We define vectors $\boldsymbol{\xi}^{(j)} \in[0,+\infty\rangle^{k}(j=1, \ldots, n+2)$ and weights $\mathbf{w}^{\prime}$ as

$$
\begin{gathered}
\boldsymbol{\xi}_{i}^{(1)}=a_{i}, \quad \xi_{i}^{(n+2)}=b_{i} \quad(i=1, \ldots, k), \\
\boldsymbol{\xi}^{(j)}=\boldsymbol{x}^{(j-1)} \quad(j=2, \ldots, n+1), \\
w_{1}^{\prime}=1, \quad w_{2}^{\prime}=-w_{1}, \ldots, w_{n+1}^{\prime}=w_{n}, \quad w_{n+2}^{\prime}=1 .
\end{gathered}
$$

Obviously, we have $\xi^{(1)} \leq \cdots \leq \boldsymbol{\xi}^{(n+2)}$ and

$$
0 \leq W_{k}^{\prime} \leq 1 \quad(k=1, \ldots, n+1), \quad W_{n+2}^{\prime}=1 .
$$

We can apply Theorem 2.3 on $\boldsymbol{\xi}^{(j)}(j=1, \ldots, n+2)$ and $\mathbf{w}^{\prime}$ to obtain

$$
\prod_{i=1}^{k} \sum_{j=1}^{n+2} w_{j}^{\prime} \xi_{i}^{(j)} \leq \sum_{j=1}^{n+2} w_{j}^{\prime n+2} w_{j}^{\prime} \prod_{i=1}^{k} \xi_{i}^{(j)}
$$

from which (2.20) immediately follows. If $\mathbf{x}^{(1)} \geq \cdots \geq \mathbf{x}^{(n)}$, the proof is similar.

\section{Variants of Pečarić's inequalities}

In 1984 Pečarić proved several generalizations of the discrete Ostrowski's inequalities. Here we give two of them which are interesting to us because they are refinements of Theorem 2.1.

Theorem 3.1 [7, Theorem 3]. Let $\mathbf{x}=\left(x_{1}, \ldots, x_{n}\right)$ and $\mathbf{y}=\left(y_{1}, \ldots, y_{n}\right)$ be real $n$-tuples monotonic in the same direction and let $\mathbf{w}=\left(w_{1}, \ldots, w_{n}\right)$ be a real $n$-tuple such that

$$
0 \leq W_{k} \leq W_{n} \quad(k=1, \ldots, n-1) .
$$

If $m$ and $r$ are nonnegative real numbers such that

$$
\left|x_{k+1}-x_{k}\right| \geq m, \quad\left|y_{k+1}-y_{k}\right| \geq r \quad(k=1, \ldots, n-1),
$$

then

$$
T(\mathbf{x}, \mathbf{y} ; \mathbf{w}) \geq m r T(\mathbf{e}, \mathbf{e} ; \mathbf{w}) \geq 0
$$


6 Variants of Čebyšev's inequality with applications

where

$$
\begin{aligned}
T(\mathbf{x}, \mathbf{y} ; \mathbf{w})= & \sum_{i=1}^{n} w_{i} \sum_{i=1}^{n} w_{i} x_{i} y_{i}-\sum_{i=1}^{n} w_{i} x_{i} \sum_{i=1}^{n} w_{i} y_{i}, \\
& \mathbf{e}=(0,1, \ldots, n-1) .
\end{aligned}
$$

If $\mathbf{x}$ and $\mathbf{y}$ are monotonic in opposite directions, then

$$
T(\mathbf{x}, \mathbf{y} ; \mathbf{w}) \leq-m r T(\mathbf{e}, \mathbf{e} ; \mathbf{w}) \leq 0
$$

Theorem 3.2 [7, Theorem 4]. Let $\mathbf{x}$ and $\mathbf{y}$ be real $n$-tuples such that

$$
\left|x_{k+1}-x_{k}\right| \leq M, \quad\left|y_{k+1}-y_{k}\right| \leq R \quad(k=1, \ldots, n-1)
$$

hold for some nonnegative real numbers $M$ and $R$, and let $\mathbf{w}$ be a real $n$-tuple such that (3.1) is valid. Then

$$
|T(\mathbf{x}, \mathbf{y} ; \mathbf{w})| \leq M R T(\mathbf{e}, \mathbf{e} ; \mathbf{w}) .
$$

In the next two theorems we give Mercer's type variants of Theorems 3.1 and 3.2 which are refinements of Theorem 2.2.

Theorem 3.3. Let $n \geq 2$ and let $\mathbf{w}$ be a real $n$-tuple such that (2.12) is valid. Let $[a, b],[c, d]$ be intervals in $\mathbb{R}$, where $a<b, c<d$. Let $\mathbf{x}=\left(x_{1}, \ldots, x_{n}\right) \in[a, b]^{n}$ and $\mathbf{y}=\left(y_{1}, \ldots, y_{n}\right) \in[c, d]^{n}$ be monotonic n-tuples, and let $m$ and $r$ be nonnegative real numbers such that

$$
\begin{array}{cc}
\min _{1 \leq i \leq n} x_{i}-a \geq m, & b-\max _{1 \leq i \leq n} x_{i} \geq m, \\
\left|x_{k+1}-x_{k}\right| \geq m & (k=1, \ldots, n-1), \\
\min _{1 \leq i \leq n} y_{i}-c \geq r, & d-\max _{1 \leq i \leq n} y_{i} \geq r, \\
\left|y_{k+1}-y_{k}\right| \geq r & (k=1, \ldots, n-1) .
\end{array}
$$

If $\mathbf{x}$ and $\mathbf{y}$ are monotonic in the same direction, then

$$
\tilde{T}(\mathbf{x}, \mathbf{y} ; \mathbf{w}) \geq m r[\tilde{T}(\mathbf{f}, \mathbf{f} ; \mathbf{w})+2 n] \geq 0,
$$

where

$$
\begin{gathered}
\tilde{T}(\mathbf{x}, \mathbf{y} ; \mathbf{w})=a c+b d-\sum_{i=1}^{n} w_{i} x_{i} y_{i}-\left(a+b-\sum_{i=1}^{n} w_{i} x_{i}\right)\left(c+d-\sum_{i=1}^{n} w_{i} y_{i}\right), \\
\mathbf{f}=(1, \ldots, n) \in[1, n]^{n} .
\end{gathered}
$$

If $\mathbf{x}$ and $\mathbf{y}$ are monotonic in opposite directions, then

$$
\tilde{T}(\mathbf{x}, \mathbf{y} ; \mathbf{w}) \leq-m r[\tilde{T}(\mathbf{f}, \mathbf{f} ; \mathbf{w})+2 n] \leq 0 .
$$


Proof. Suppose that $n$-tuples $\mathbf{x}$ and $\mathbf{y}$ are both monotonically decreasing (if $\mathbf{x}$ and $\mathbf{y}$ are monotonically increasing, the proof is similar). We define $(n+2)$-tuples $\mathbf{w}^{\prime}=\left(w_{1}^{\prime}, \ldots\right.$, $\left.w_{n+2}^{\prime}\right), \mathbf{x}^{\prime}=\left(x_{1}^{\prime}, \ldots, x_{n+2}^{\prime}\right)$, and $\mathbf{y}^{\prime}=\left(y_{1}^{\prime}, \ldots, y_{n+2}^{\prime}\right)$ as

$$
\begin{aligned}
& w_{1}^{\prime}=1, \quad w_{2}^{\prime}=-w_{1}, \ldots, w_{n+1}^{\prime}=-w_{n}, \quad w_{n+2}^{\prime}=1, \\
& x_{1}^{\prime}=b, \quad x_{2}^{\prime}=x_{1}, \ldots, x_{n+1}^{\prime}=x_{n}, \quad x_{n+2}^{\prime}=a, \\
& y_{1}^{\prime}=d, \quad y_{2}^{\prime}=y_{1}, \ldots, y_{n+1}^{\prime}=y_{n}, \quad y_{n+2}^{\prime}=c \text {. }
\end{aligned}
$$

Obviously, $\mathbf{x}^{\prime}$ and $\mathbf{y}^{\prime}$ are both monotonically decreasing and we have

$$
\begin{gathered}
0 \leq W_{k}^{\prime} \leq 1 \quad(k=1, \ldots, n+1), \quad W_{n+2}^{\prime}=1, \\
\left|x_{k+1}^{\prime}-x_{k}^{\prime}\right| \geq m, \quad\left|y_{k+1}^{\prime}-y_{k}^{\prime}\right| \geq r \quad(k=1, \ldots, n+1) .
\end{gathered}
$$

From Theorem 3.1 we have

$$
T\left(\mathbf{x}^{\prime}, \mathbf{y}^{\prime} ; \mathbf{w}^{\prime}\right) \geq \operatorname{mr} T\left(\mathbf{e}^{\prime}, \mathbf{e}^{\prime} ; \mathbf{w}^{\prime}\right) \geq 0,
$$

where

$$
\mathbf{e}^{\prime}=(0,1, \ldots, n+1)
$$

From that we immediately obtain

$$
\begin{aligned}
& a c+b d-\sum_{i=1}^{n} w_{i} x_{i} y_{i}-\left(a+b-\sum_{i=1}^{n} w_{i} x_{i}\right)\left(c+d-\sum_{i=1}^{n} w_{i} y_{i}\right) \\
& \geq m r\left[\sum_{i=1}^{n+2} w_{i}^{\prime}(i-1)^{2}-\left(\sum_{i=1}^{n+2} w_{i}^{\prime}(i-1)\right)^{2}\right] \\
&=m r\left[(n+1)^{2}-\sum_{i=1}^{n} w_{i} i^{2}-\left(n+1-\sum_{i=1}^{n} w_{i} i\right)^{2}\right] \geq 0,
\end{aligned}
$$

that is,

$$
\tilde{T}(\mathbf{x}, \mathbf{y} ; \mathbf{w}) \geq \operatorname{mr}[\tilde{T}(\mathbf{f}, \mathbf{f} ; \mathbf{w})+2 n] \geq 0
$$

If $n$-tuples $\mathbf{x}$ and $\mathbf{y}$ are monotonic in opposite directions, the proof is similar.

Theorem 3.4. Let $n \geq 2$ and let $\mathbf{w}$ be a real $n$-tuple such that $(2.12)$ is valid. Let $[a, b],[c, d]$ be intervals in $\mathbb{R}$, where $a<b, c<d$. Let $\mathbf{x}=\left(x_{1}, \ldots, x_{n}\right) \in[a, b]^{n}, \mathbf{y}=\left(y_{1}, \ldots, y_{n}\right) \in[c, d]^{n}$ and let $M$ and $R$ be nonnegative real numbers such that

$$
\begin{array}{ll}
\left|x_{1}-a\right| \leq M, & \left|b-x_{n}\right| \leq M, \\
\left|x_{k+1}-x_{k}\right| \leq M & (k=1, \ldots, n-1), \\
\left|y_{1}-c\right| \leq R, & \left|d-y_{n}\right| \leq R, \\
\left|y_{k+1}-y_{k}\right| \leq R & (k=1, \ldots, n-1) .
\end{array}
$$


8 Variants of Čebyšev's inequality with applications

Then

$$
|\tilde{T}(\mathbf{x}, \mathbf{y} ; \mathbf{w})| \leq M R[\tilde{T}(\mathbf{f}, \mathbf{f} ; \mathbf{w})+2 n] \leq 0 .
$$

Proof. Similarly as in Theorem 3.3.

Corollary 3.5. Let $n \geq 2$ and let $[a, b]$ be an interval in $\mathbb{R}$ where $a<b$. Then for all $\mathbf{x}=$ $\left(x_{1}, \ldots, x_{n}\right) \in[a, b]^{n}$,

$$
\left[n a^{2}+n b^{2}-\sum_{i=1}^{n} x_{i}^{2}-\frac{1}{n}\left(n a+n b-\sum_{i=1}^{n} x_{i}\right)^{2}\right] \frac{12}{n(n+1)(5 n+7)} \geq m^{2}
$$

where

$$
m=\min _{0 \leq i<j \leq n+1}\left|x_{i}-x_{j}\right|, \quad x_{0}=a, \quad x_{n+1}=b .
$$

Proof. Directly from Theorem 3.3.

Corollary 3.6. Let $\mathbf{x}=\left(x_{1}, \ldots, x_{n}\right), \mathbf{y}=\left(y_{1}, \ldots, y_{n}\right), M$ and $R$ be defined as in Theorem 3.4. Then

$$
\left|n a c+n b d-\sum_{i=1}^{n} x_{i} y_{i}-\frac{1}{n}\left(n a+n b-\sum_{i=1}^{n} x_{i}\right)\left(n c+n d-\sum_{i=1}^{n} y_{i}\right)\right| \leq \frac{n(n+1)(5 n+7)}{12} M R .
$$

Proof. Directly from Theorem 3.4.

The above results are variants of some Lupaş' results [3].

\section{Applications: inequality of Milne and its converse}

In 1925 Milne [5] obtained the following interesting integral inequality for positive functions $f$ and $g$ which are integrable on $[a, b]$ :

$$
\int_{a}^{b} \frac{f(x) g(x)}{f(x)+g(x)} d x \int_{a}^{b}[f(x)+g(x)] d x \leq \int_{a}^{b} f(x) d x \int_{a}^{b} g(x) d x .
$$

In 2000 Rao [9] combined Milne's inequality and the well-known inequality between arithmetic and geometric means to obtain the following double inequality for sums.

Proposition 4.1. Let $n \geq 2$ and let $w_{i}>0(i=1,2, \ldots, n)$ be real numbers with $\sum_{i=1}^{n} w_{i}=1$. Then for all real numbers $p_{i} \in\langle-1,1\rangle(i=1, \ldots, n)$,

$$
\sum_{i=1}^{n} \frac{w_{i}}{1-p_{i}^{2}} \leq\left(\sum_{i=1}^{n} \frac{w_{i}}{1-p_{i}}\right)\left(\sum_{i=1}^{n} \frac{w_{i}}{1+p_{i}}\right) \leq\left(\sum_{i=1}^{n} \frac{w_{i}}{1-p_{i}^{2}}\right)^{2}
$$

Two years later Alzer and Kovačec obtained the following refinement of (4.2). 
Theorem 4.2 [2, Theorem 1 ]. Let $n \geq 2$ and let $w_{i}>0(i=1,2, \ldots, n)$ be real numbers with $\sum_{i=1}^{n} w_{i}=1$. Then for all real numbers $p_{i} \in[0,1\rangle(i=1, \ldots, n)$,

$$
\left(\sum_{i=1}^{n} \frac{w_{i}}{1-p_{i}^{2}}\right)^{\alpha} \leq\left(\sum_{i=1}^{n} \frac{w_{i}}{1-p_{i}}\right)\left(\sum_{i=1}^{n} \frac{w_{i}}{1+p_{i}}\right) \leq\left(\sum_{i=1}^{n} \frac{w_{i}}{1-p_{i}^{2}}\right)^{\beta}
$$

with the best possible exponents

$$
\alpha=1, \quad \beta=2-\min _{1 \leq i \leq n} w_{i}
$$

We note here that the crucial step in the proof of Theorem 4.2 was performed by using a discrete variant of the Čebyšev's inequality (see, e.g., [8, page 197]) which itself was generalized in Section 2. This enables us to give the following generalization of Theorem 4.2.

Theorem 4.3. Let $n \geq 2$ and let $\mathbf{w}=\left(w_{1}, \ldots, w_{n}\right)$ be a real $n$-tuple such that $(2.12)$ is satisfied. Then for all $\alpha \in\langle-\infty, 1], \beta \in\left[2-\min _{1 \leq i \leq n} W_{i},+\infty\right\rangle$ and for all monotonic $n$-tuples $\mathbf{p}=\left(p_{1}, \ldots, p_{n}\right) \in[0,1\rangle^{n}$,

$$
\left(\sum_{i=1}^{n} \frac{w_{i}}{1-p_{i}^{2}}\right)^{\alpha} \leq\left(\sum_{i=1}^{n} \frac{w_{i}}{1-p_{i}}\right)\left(\sum_{i=1}^{n} \frac{w_{i}}{1+p_{i}}\right) \leq\left(\sum_{i=1}^{n} \frac{w_{i}}{1-p_{i}^{2}}\right)^{\beta}
$$

with the best possible exponents

$$
\alpha=1, \quad \beta=2-\min _{1 \leq i \leq n} W_{i} .
$$

Proof. We follow the idea of the proof given in [2]. Suppose that $1>p_{1} \geq p_{2} \geq \cdots \geq$ $p_{n} \geq 0$. It can be easily seen that

$$
\begin{aligned}
& 0< \frac{1}{1+p_{1}} \leq \frac{1}{1+p_{2}} \leq \cdots \leq \frac{1}{1+p_{n}} \leq 1, \\
& \frac{1}{1-p_{1}} \geq \frac{1}{1-p_{2}} \geq \cdots \geq \frac{1}{1-p_{n}} \geq 1, \\
& \frac{1}{1-p_{1}^{2}} \geq \frac{1}{1-p_{2}^{2}} \geq \cdots \geq \frac{1}{1-p_{n}^{2}} \geq 1,
\end{aligned}
$$

so in this case (see Remark 1.3) we know that

$$
\sum_{i=1}^{n} \frac{w_{i}}{1-p_{i}} \geq 1, \quad \sum_{i=1}^{n} \frac{w_{i}}{1+p_{i}}>0, \quad \sum_{i=1}^{n} \frac{w_{i}}{1-p_{i}^{2}} \geq 1 .
$$

Let $w=\min _{1 \leq i \leq n} W_{i}$. We define function $f:[0,1\rangle^{n} \rightarrow \mathbb{R}$ as

$$
f\left(p_{1}, \ldots, p_{n}\right)=(2-w) \log \left(\sum_{i=1}^{n} \frac{w_{i}}{1-p_{i}^{2}}\right)-\log \left(\sum_{i=1}^{n} \frac{w_{i}}{1-p_{i}}\right)-\log \left(\sum_{i=1}^{n} \frac{w_{i}}{1+p_{i}}\right) .
$$

For fixed $k \in\{1, \ldots, n-1\}$ we define function $f_{k}:[0,1\rangle \rightarrow \mathbb{R}$ as

$$
f_{k}(p)=f\left(p, \ldots, p, p_{k+1}, \ldots, p_{n}\right) .
$$


Let $p \in\left[p_{k+1}, 1\right\rangle$. We have

$$
f_{k}^{\prime}(p)=\frac{W_{k} D}{\left(1-p^{2}\right) A B C}
$$

where

$$
\begin{gathered}
A=W_{k}+\sum_{i=k+1}^{n} w_{i} \frac{1-p^{2}}{1-p_{i}^{2}}, \quad B=W_{k}+\sum_{i=k+1}^{n} w_{i} \frac{1-p}{1-p_{i}}, \quad C=W_{k}+\sum_{i=k+1}^{n} w_{i} \frac{1+p}{1+p_{i}}, \\
D=A[(1-p) B-(1+p) C]+2(2-w) p B C .
\end{gathered}
$$

We define $n$-tuples $\mathbf{x}=\left(x_{1}, \ldots, x_{n}\right)$ and $\mathbf{y}=\left(y_{1}, \ldots, y_{n}\right)$ with

$$
\begin{gathered}
x_{i}=1, \quad y_{i}=1 \quad(i=1, \ldots, k), \\
x_{i}=\frac{1-p}{1-p_{i}}, \quad y_{i}=\frac{1+p}{1+p_{i}} \quad(i=k+1, \ldots, n),
\end{gathered}
$$

which are obviously monotonic in opposite directions. From Theorem 2.1 we have

$$
\left(\sum_{i=1}^{n} w_{i} x_{i}\right)\left(\sum_{i=1}^{n} w_{i} y_{i}\right) \geq \sum_{i=1}^{n} w_{i} x_{i} y_{i}
$$

that is, $B C \geq A$, and from Remark 1.3 we know that $A, B$, and $C$ are all positive. This enables us to conclude that

$$
\begin{aligned}
\frac{D}{A} & \geq(1-p) B-(1+p) C+2(2-w) p \\
& =2 p\left(2-w-W_{k}\right)+\sum_{i=k+1}^{n} w_{i}\left(\frac{(1-p)^{2}}{1-p_{i}}-\frac{(1+p)^{2}}{1+p_{i}}\right) .
\end{aligned}
$$

It can be easily seen that

$$
\begin{aligned}
-4 p & =(1-p)^{2}-(1+p)^{2} \leq \frac{(1-p)^{2}}{1-p_{k+1}}-\frac{(1+p)^{2}}{1+p_{k+1}} \\
& \leq \cdots \leq \frac{(1-p)^{2}}{1-p_{n}}-\frac{(1+p)^{2}}{1+p_{n}}
\end{aligned}
$$

so we have

$$
\sum_{i=1}^{k} w_{i}(-4 p)+\sum_{i=k+1}^{n} w_{i}\left(\frac{(1-p)^{2}}{1-p_{i}}-\frac{(1+p)^{2}}{1+p_{i}}\right) \geq-4 p
$$

that is,

$$
\sum_{i=k+1}^{n} w_{i}\left(\frac{(1-p)^{2}}{1-p_{i}}-\frac{(1+p)^{2}}{1+p_{i}}\right) \geq-4 p+4 p W_{k}
$$


From (4.19) and (4.16) we obtain

$$
\frac{D}{A} \geq 2 p\left(W_{k}-w\right) \geq 0
$$

which implies that the function $f_{k}$ is increasing on $\left[p_{k+1}, 1\right\rangle$. Using that fact we obtain

$$
\begin{aligned}
f\left(p_{1}, \ldots, p_{n}\right) & =f_{1}\left(p_{1}\right) \geq f_{1}\left(p_{2}\right)=f_{2}\left(p_{2}\right) \geq f_{2}\left(p_{3}\right)=f_{3}\left(p_{3}\right) \\
& \geq \cdots \geq f_{n-1}\left(p_{n}\right)=-(1-w) \log \left(1-p_{n}^{2}\right) \geq 0,
\end{aligned}
$$

which implies

$$
\left(\sum_{i=1}^{n} \frac{w_{i}}{1-p_{i}}\right)\left(\sum_{i=1}^{n} \frac{w_{i}}{1+p_{i}}\right) \leq\left(\sum_{i=1}^{n} \frac{w_{i}}{1-p_{i}^{2}}\right)^{2-w},
$$

that is, the right inequality in (4.5) holds for $\beta=2-\min _{1 \leq i \leq n} W_{i}$. Since

$$
\sum_{i=1}^{n} \frac{w_{i}}{1-p_{i}^{2}} \geq 1
$$

it is clear that it also holds for all $\beta \geq 2-\min _{1 \leq i \leq n} W_{i}$.

A similar argument as in [2] shows that $\beta=2-\min _{1 \leq i \leq n} W_{i}$ gives the best upper bound in (4.5): if $W_{k}=\min _{1 \leq i \leq n} W_{i}$, we simply choose $n$-tuple $\mathbf{p}=\left(p_{1}, \ldots, p_{n}\right)$ defined as

$$
p_{1}=\cdots=p_{k}=q, \quad p_{k+1}=\cdots=p_{n}=0, \quad q \in\langle 0,1\rangle,
$$

and for such $\mathbf{p}$ and $\mathbf{w}$ we obtain that $\beta$ must satisfy the condition $\beta \geq 2-W_{k}$.

The left-hand side of (4.5) is a simple consequence of Theorem 2.1. If we define

$$
x_{i}=\frac{1}{1-p_{i}}, \quad y_{i}=\frac{1}{1+p_{i}} \quad(i=1, \ldots, n)
$$

then $n$-tuples $\mathbf{x}=\left(x_{1}, \ldots, x_{n}\right)$ and $\mathbf{y}=\left(y_{1}, \ldots, y_{n}\right)$ are monotonic in opposite directions, so we have

$$
\sum_{i=1}^{n} \frac{w_{i}}{1-p_{i}^{2}} \leq\left(\sum_{i=1}^{n} \frac{w_{i}}{1-p_{i}}\right)\left(\sum_{i=1}^{n} \frac{w_{i}}{1+p_{i}}\right) .
$$

Furthermore, (4.23) implies

$$
\left(\sum_{i=1}^{n} \frac{w_{i}}{1-p_{i}^{2}}\right)^{\alpha} \leq\left(\sum_{i=1}^{n} \frac{w_{i}}{1-p_{i}}\right)\left(\sum_{i=1}^{n} \frac{w_{i}}{1+p_{i}}\right)
$$

for all $\alpha \leq 1$. 
The same argument as in [2] shows that $\alpha=1$ gives the best lower bound for (4.5). In case $0 \leq p_{1} \leq \cdots \leq p_{n}<1$ the proof is similar.

In the next theorem we give a Mercer's type variant of (4.5).

Theorem 4.4. Let $n \geq 2$ and let $\mathbf{w}=\left(w_{1}, \ldots, w_{n}\right)$ be a real $n$-tuple such that (2.12) is satisfied. Then for all $\alpha \in\langle-\infty, 1], \beta \in[2,+\infty\rangle$ and for all monotonic $n$-tuples $\mathbf{p}=\left(p_{1}, \ldots, p_{n}\right) \in$ $[p, q]^{n}$, where $[p, q] \subseteq[0,1\rangle$ and $p<q$,

$$
\begin{aligned}
& \left(\frac{1}{1-p^{2}}+\frac{1}{1-q^{2}}-\sum_{i=1}^{n} \frac{w_{i}}{1-p_{i}^{2}}\right)^{\alpha} \\
& \quad \leq\left(\frac{1}{1-p}+\frac{1}{1-q}-\sum_{i=1}^{n} \frac{w_{i}}{1-p_{i}}\right)\left(\frac{1}{1+p}+\frac{1}{1+q}-\sum_{i=1}^{n} \frac{w_{i}}{1+p_{i}}\right) \\
& \quad \leq\left(\frac{1}{1-p^{2}}+\frac{1}{1-q^{2}}-\sum_{i=1}^{n} \frac{w_{i}}{1-p_{i}^{2}}\right)^{\beta},
\end{aligned}
$$

with the best possible exponents

$$
\alpha=1, \quad \beta=2
$$

Proof. Suppose that $q \geq p_{1} \geq p_{2} \geq \cdots \geq p_{n} \geq p$. We define $(n+2)$-tuples $\mathbf{w}^{\prime}=\left(w_{1}^{\prime}, \ldots\right.$, $\left.w_{n+2}^{\prime}\right)$ and $\mathbf{p}^{\prime}=\left(p_{1}^{\prime}, \ldots, p_{n+2}^{\prime}\right) \in[0,1\rangle^{n}$ with

$$
\begin{gathered}
w_{1}^{\prime}=1, \quad w_{2}^{\prime}=-w_{1}, \ldots, w_{n+1}^{\prime}=-w_{n}, \quad w_{n+2}^{\prime}=1, \\
p_{1}^{\prime}=q, \quad p_{2}^{\prime}=p_{1}, \ldots, p_{n+1}^{\prime}=p_{n}, \quad p_{n+2}^{\prime}=p .
\end{gathered}
$$

We have

$$
0 \leq W_{k}^{\prime} \leq 1 \quad(k=1, \ldots, n+1), \quad W_{n+2}^{\prime}=1, \quad \min _{1 \leq i \leq n} W_{i}^{\prime}=0 .
$$

From Remark 1.3 we know that

$$
\sum_{i=1}^{n+2} \frac{w_{i}^{\prime}}{1-p_{i}^{\prime 2}} \geq 1
$$

so the left side and the right side of (4.28) are well defined. If we apply Theorem 4.3 on $(n+2)$-tuples $\mathbf{w}^{\prime}$ and $\mathbf{p}^{\prime}$, we obtain

$$
\left(\sum_{i=1}^{n+2} \frac{w_{i}^{\prime}}{1-p_{i}^{\prime 2}}\right)^{\alpha} \leq\left(\sum_{i=1}^{n+2} \frac{w_{i}^{\prime}}{1-p_{i}^{\prime}}\right)\left(\sum_{i=1}^{n+2} \frac{w_{i}^{\prime}}{1+p_{i}^{\prime}}\right) \leq\left(\sum_{i=1}^{n+2} \frac{w_{i}^{\prime}}{1-p_{i}^{\prime 2}}\right)^{\beta},
$$

from which (4.28) immediately follows.

If $p \leq p_{1} \leq \cdots \leq p_{n} \leq q$, the proof is similar. 


\section{References}

[1] S. Abramovich, M. Klaričić Bakula, M. Matić, and J. Pečarić, A variant of Jensen-Steffensen's inequality and quasi-arithmetic means, Journal of Mathematical Analysis and Applications 307 (2005), no. 1, 370-386.

[2] H. Alzer and A. Kovačec, The inequality of Milne and its converse, Journal of Inequalities and Applications 7 (2002), no. 4, 603-611.

[3] A. Lupaş, On an inequality, Publikacije Elektrotehnickog Fakulteta Univerziteta U Beogradu. Serija Matematika i Fizika (1981), no. 716-734, 32-34.

[4] A. McD. Mercer, A variant of Jensen's inequality, Journal of Inequalities in Pure and Applied Mathematics 4 (2003), no. 4, 1-2, article 73.

[5] E. A. Milne, Note on Rosseland's integral for the stellar absorption coefficient, Monthly Notices of the Royal Astronomical Society 85 (1925), 979-984.

[6] J. Pečarić, On the Čebyšev inequality, Buletinul Ştiinţific şi Tehnic Institutului Politehnic "Traian Vuia” Timişoara 25(39) (1980), no. 1, 5-9 (1981).

[7] _ On the Ostrowski generalization of Čebyšev's inequality, Journal of Mathematical Analysis and Applications 102 (1984), no. 2, 479-487.

[8] J. Pečarić, F. Proschan, and Y. L. Tong, Convex Functions, Partial Orderings, and Statistical Applications, Mathematics in Science and Engineering, vol. 187, Academic Press, Massachusetts, 1992.

[9] C. R. Rao, Statistical proofs of some matrix inequalities, Linear Algebra and Its Applications 321 (2000), no. 1-3, 307-320.

M. Klaričić Bakula: Department of Mathematics, Faculty of Natural Sciences, Mathematics, and Education, University of Split, Teslina 12, 21000 Split, Croatia

E-mail address: milica@pmfst.hr

A. Matković: Department of Mathematics, Faculty of Natural Sciences, Mathematics, and Education, University of Split, Teslina 12, 21000 Split, Croatia

E-mail address: anita@pmfst.hr

J. Pečarić: Faculty of Textile Technology, University of Zagreb, Pierottijeva 6, 10000 Zagreb, Croatia E-mail address: pecaric@hazu.hr 